University for Business and Technology in Kosovo

UBT Knowledge Center

Oct 27th, 9:00 AM - 10:30 AM

\title{
Impact of Psychosocial Factors on Postpartum Depression of Mothers
}

Anita Sadikaj

University for Business and Technology, anita.sadikaj@hotmail.com

Artemisi Shehu

University of Tirana

Follow this and additional works at: https://knowledgecenter.ubt-uni.net/conference

Part of the Medicine and Health Sciences Commons

\section{Recommended Citation}

Sadikaj, Anita and Shehu, Artemisi, "Impact of Psychosocial Factors on Postpartum Depression of Mothers" (2018). UBT International Conference. 364.

https://knowledgecenter.ubt-uni.net/conference/2018/all-events/364

This Event is brought to you for free and open access by the Publication and Journals at UBT Knowledge Center. It has been accepted for inclusion in UBT International Conference by an authorized administrator of UBT Knowledge Center. For more information, please contact knowledge.center@ubt-uni.net. 


\title{
Impact of Psychosocial Factors on Postpartum Depression of Mothers
}

\author{
Anita Sadikaj ${ }^{1}$, Artemisi Shehu ${ }^{2}$ \\ ${ }^{1}$ UBT-Higher Education Institution \\ ${ }^{2}$ DPP, Faculty of Social Sciences, University of Tirana \\ 1anita.sadikaj@hotmail.com; \\ artemisashehu@yahoo.com
}

\begin{abstract}
Postpartum depression (PPD) is an important depression episode which begins in pregnancy or within 4 weeks after giving birth. Research is conducted in Gynecology and Obstetrics Clinic, Pediatric Clinic and in private Hospital "Lindja", in Prishtina, during period May-June in 2018. Study is focused in relation of psychosocial factors such as: birthplace, education, employment, economic level, mental health, number of children, breastfeeding, anxiety, spousal relationship and social support. Subjects of this study were 136 mothers, from the third day of giving birth till one year after this. The instruments were self-reporters, using Edinburgh Postnatal Depression Scale (EPDS). Results showed that prevalence of depression was $12,61 \%$, in the sample of 136 subjects with an average age of $31.3 \pm 6.4$, years of education $12 \pm 2,5$, mostly living in rural area $44.9 \%$. Related to social support, mothers report high level of family and spousal support (respectively $\mathrm{M}=22.13 \pm 5.5 ; \mathrm{M}=22.78 \pm 5.46$ ) and lower level of social support. Results notify that most of these factors have significant role.
\end{abstract}

Keywords: Mothers, PPD, Psychosocial factors, Family support

\section{Introduction}

The postnatal period is known as the time when women develop susceptibility to a variety of emotional symptoms. However, postpartum depression is widely considered as a possible mood disorder.

The American Psychological Association describes postpartum depression as, "a serious mental health problem characterized by a prolonged period of emotional disturbance, occurring at a time of major life change and increased responsibilities in the care of a newborn infant" (APA, 2015). Postpartum Depression is the most emotional problem spread throughout women's lives (Guardino \& Schetter, 2014). PPD 
is an easily recognizable, very widespread but very underdeveloped disease. Previous studies on different communities show that mental disorders in women are mainly seen in reproductive age, whereas after childbirth the risks of emerging of these problems have increased (Oslen et al, 2009). Therefore, pregnancy and motherhood are known as a possible period of women's mental health vulnerability.

Epidemiological studies generally show that the prevalence of postpartum depresion is in the range of 10-20\% (Appleby et al., 1997; Warner et al., 1996; Swain, O'Hara, Starr and Gorman, 1997; Brugha et al, 1998; Lee et al, 2001; Chandran et al, 2002; Wisner et al,2014; Chen et al, 2018)

Postpartum depression typically begins before or any time after childbirth, but generally develops between one week and one month after delivery. Mothers experiencing postpartum depression describe feelings of extreme sadness, anxiety, and exhaustion (Thurgood, 2009; National Institute of Medicine, 2015)

Previous research has identified risk factors that can increase a woman's chance of developing postpartum depression. If there is a history of mental health disorders during or after a previous pregnancy, previous experience with mental health disorder, and a family history of depression or other mental health illnesses increases the chances of a mother developing postpartum depression (Vigot et al., 2010).

Also, Laggford, noted a lack of strong emotional support from a spouse, partner, family, or friends has also been significant in determining the development of postpartum depression (Laggford, 2009). In addition medical complications during the childbirth proces or mixed feeling about the pregnancy have been found to increase the chances of a woman developing PPD. Despite the efforts of researchers, the etiology of postpartum depresion is unstable and unknown (Lusskin et al, 2007).

Beck (1967) postulated that depressed mood is the result of thought disturbances. Pessimism toward oneself, the world, and the future contributes to a depressive mood. In addition, in the absence of suitable role models, the woman feels loss of control and anxiety resulting in a lack of a capability to cope with infant's demands and care (Nemade et al, 2011). Some experts have also noted that childbirth results in the loss of their identity and leads to the attraction of love and loss of independence (Kaplan \& Sadock, 2010). Moreover, the negative attitude of the family affects the well-being of women and results in non-adaptation of the coping mechanisms.

If PPD is not treated, it may last for a period of time, often months or years, sometimes becoming a chronic depressive disorder. Untreated postpartum depresion may have effects on child, infants, fetuses and mothers (Abdollahi \& Zarghami,2016).

Referring to the above studies can be said that PPD is a phenomenon that is present in many women's cases, but less studied. This phenomen varies from one culture to another. It is influenced by many psychological, interpersonal, social and cultural factors. Among the factors influencing the development of PP are genetic history of the case, previous experience with mental health disorder and a family history of depresion or other mental health disorders, birth problems, and many more. 


\section{Methods}

The aim of this study was to assess impact of psychological factors during the postpartum depression. Study is focused in relation of psychosocial factors such as: birthplace, education, employment, economic level, mental health, number of children, breastfeeding, anxiety, spousal relationship and social support.

The study has prospective design, with a single sampling phase. Parts of this study were women who gave birth between May-June 2018, at Clinic of Gynecology and Obstetrics, Pediatric Clinic at University Clinical Center of Kosova and in private Gynecological Hospital "Lindja", in Prishtina. In total they where 136 woman's who were hospitalized from the third day of giving birth till one year after this. These mothers were from different settlements in Kosovo and age ranged from 16 to 55 years, all of them where interwiew by authors of this study and lasted between 20 minutes and 50 minutes.

Ethics approval for the study was granted by the Ethics Committee of University Clinical Centre of Kosova. Participants were informed about the objectives of the survey and ensured anonymity. All participants provided written informed consent. Only women who were willing to participate in the survey were interviewed and completed self-reporting questionnaires.

In the first part questionnaire was completed with questions on socio-demographic data for research interests, such as the number of pregnancies, delivery and newborn, breastfeeding, childcare and family support, relationship with partner, family status, social status, residence, working and economic status, age, data on somatic diseases and mental illnesses in family history, previous mental illnesses, especially in pregnancies and previous births, etc.

The second part was the Edinburgh Postnatal Depression Scale (EPDS) an internationally used ten-item self-report questionnaire, designed as a screening tool to identify depressive symptoms in the perinatal period. Items of the scale correspond to various clinical depression symptoms, such as guilt feeling, sleep disturbance, low energy, anhedonia, and suicidal ideation.

EPDS questionnaire is used in a significant number of research works and reliability and validity are well documented. The overall reliability (Cronbach's alpha) of EPDS at our study was $\alpha=.846$.

\section{Results and discussions}

Results showed that prevalence of depression was an increasing indicator $(M=12.61$; $\mathrm{SD}=6.67$ ) in the sample of 136 subjects with an average age $31.3 \pm 6.4$, years of education $12 \pm 2,5$, mostly living in urban area $55.1 \%$, and underwent caesarean section delivery in $49.3 \%$ of cases. Referring to the economic status $66.2 \%$ reported the middle level. In the respondents' households, $19.1 \%$ reported being suffering from depression and $17.6 \%$ of mothers suffered from a chronic illness.be used to emphasize words in running text. Bold type and underlining should be avoided. 
According to the questions about depression, filled out by all women in the sample $40.4 \%$ responded positively to the question of loss of interest and satisfaction for life. None of them did not attend any professional treatment, which requires a more serious approach to this problem.

Table 1. Correlation for postpartum depression

\begin{tabular}{ll}
\hline Factors & Correlation \\
\hline $\begin{array}{l}\text { Earliest birth } \\
\text { experience }\end{array}$ & $.180^{*}$ \\
$\begin{array}{l}\text { Delivery } \\
\text { complication } \\
\text { Delivery mode }\end{array}$ & $.243^{* *}$ \\
Breastfeeding & .158 \\
Social support & $-.259^{*}$ \\
Family support & $-.534^{* *}$ \\
Anxiety & $-.504^{* *}$ \\
\hline Note ${ }^{*}$. Correlation is significant at 0.05 \\
${ }^{* *}$. Correlation is significant at 0.01
\end{tabular}

Correlational analysis between postpartum depression measured by EPDS and early birth experience, delivery complications and difficulties, delivery mode, breastfeeding, anxiety and social and family support, showed that there are important statistical links.

Previous delivery is significantly associated with PPD. Also, difficult maternal experiences according to the correlational analysis, appear to have significant statistical correlation $(r=-.243 ; \mathrm{p}<0.01)$ which means the harder the birth is, the higher postpartum depression rate reported by the mothers. We did not find any correlation between delivery mode and PPD ( $r=.158)$.

Breastfeeding seems to be a very important on increasing PPD of mothers. Shows that there is a negative significant correlation $(r=-.259 ; \mathrm{p}<0.01)$. Research has been showing that breastfeeding promotes hormonal and psychological conditions and processes that are inversely associated with postpartum depression. However, the simultaneous study of these dimensions and their potential explanatory value in the connection between breastfeeding and pre- and postpartum depression has not yet been accomplished.

Family and social support is significantly associated with PPD $\left(r=-.504^{* *} ; p<0.01\right.$ : $\mathrm{r}=-.531 ; \mathrm{p}<0.01)$.

Attachment theory says that interpersonal struggles in an individual's life have significant influences on mental health. It is obvious that an individual requires affection which needs to be fulfilled in the initial stage of a relationship. Uncertainties concerning a relationship may result to disappointment and bring about depression and anxiety (Grupe \& Nitschke, 2013). A number of interpersonal factors play a role in women's distress and sensitivity makes them prone to develop postpartum disorders, these include insufficient social support and marital conflict. Childbirth is a significant transitional event in life and support at this stage can potentially affect women's mental status after delivery (Hunker et al, 2009). Sudden psychosocial fluctuations whithin motherhood and its challenges coupled with stresses could be other factor that may trigger PPD. Previous studies in the field of medical social work 
have revealed that postpartum depression is a global phenomenon that continues to be stigmatizing among new mothers often because mothers suffer in silence (KantrowitzGordon, 2013). A lack of strong emotional support from a spouse, partner, family, or friends has also been significant in determining the development of postpartum depresion.

Table 2: Child age and level of PPD

\begin{tabular}{lccc}
\hline & & Edinburgh Scale \\
& $0-10$ & $11-30$ \\
\hline \multirow{3}{*}{ Child age } & $0-1$ month & $24.3 \%$ & $32.4 \%$ \\
& $1-6$ month & $8.8 \%$ & $19.1 \%$ \\
& $6-12$ month & $6.6 \%$ & $8.8 \%$ \\
\hline
\end{tabular}

Based on the data analysis performed, it is clear that the percentage of depressed subjects in the mothers' group who have children a month age is higher than the percentage of other groups with $32.4 \%$, then falls between 1-6 months with $19.1 \%$, while $8.8 \%$ in the 6-12 months after birth, showing that depressive symptoms are most common in mothers of children aged 0-1 months. as the child age increases, depression decreases.

From the table analysis, it can be seen that with childbearing age the postpartum depression decreases. This can also be supported by biological factors such as rapid changes in hormone levels such as estrogen, progesterone, beta-endorphin, human chorionic gonadotropin, rapid change in cortisol concentrations (increasing during pregnancy and significant decline after delivery). Consequently, the mother becomes increasingly indifferent to her child and may show more aggressive behavior and less time for parenting.

Table 3: Correlation between education and economic level on PPD

\begin{tabular}{ll}
\hline Level & Correlation \\
\hline Education & $-.449^{* *}$ \\
Economic & $-.219^{*}$ \\
\hline Note: *. Correlation is significant at 0.05 \\
${ }^{* *}$. Correlation is significant at 0.01
\end{tabular}

In both cases we have a low negative correlation, where we see the lower level of education $(r=-.449 ; p<0.0)$ and economic status $(r=-.219 ; p<0.05)$ have impact on increasing level of PPD. Research has shown that PPD appears up to four times more often to women living in poverty, compared to women with medium and high economic status. Often it is thought that socio-economic status is the most frequent factor for PPD development. 
Table 4: The impact of the profession on PPD

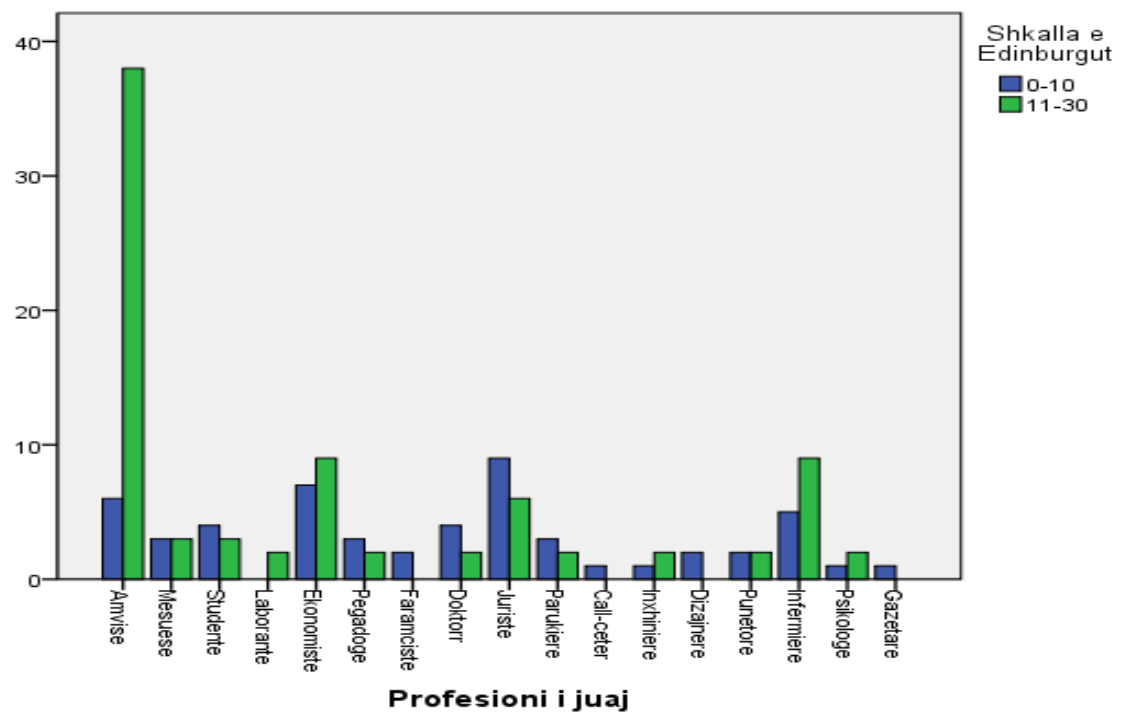

Household mothers have reported higher level of postpartum depresion, followed by nurses and economists. This may be the result of long stay in home and indor environments. So even most of the working women may not have been educated highly enough for their employment status to have a positive effect on their mental health.

Results notify that most of these factors have significant role. Factors such as education, employment, economic level, pregnancy progress and birth, children age, breastfeeding, anxiety, spousal relationship seems to have high percentage of impact in postpartum depression.

\section{Conclusions}

Our study may be helpful in better understanding of female psychosocial problems and family support after delivery and can help for the prospective planning of preventive intervention strategies in improving women's mental health.

Depression after giving birth is rising as a problem of mental health, and has negative impact on personal, family and national level.

During the research was noted that psychosocial factors are really important to preserve emotional, cognitive and behavior equilibrium.

Meanwhile family support is an important homeostatic component. Getting social help, especially after giving birth, is important to lower the risk of PPD. 


\section{References}

Abdollahi., F., Lye., M.S., \& Zarghami., M.: Perspective of postpartum depression theories: A narrative literature review (2016) Medknow Publications and Media Pvt. Ltd

American Psychological Association, A. (2015) Postpartum Depression. Retrieved from http://www.apa.org/pi/women/programs/depression/postpartum.aspx.

Appleby, L., Warner, R., Whitton, A., \& Faragher, B.: A controlled study of fluoxetine and cognitive-behavioural counselling in the treatment of postnatal depression. BMJ, (1997) 314(7085):932936

Beck AT. Depression: Clinical, experimental, and theoretical aspects. New York: Harper and Row; (1967)

Beck CT.: Theoretical perspectives of postpartum depression and their treatment implications. Am J Maternal Child Nurs (2002) 27:282-7

Chen, L., Ding, L., Qi, M., Jiang, Ch., X.-M.M., Cai, W-Z.: Incidence of and social-demographic and obstetric factors associated with postpartum depression: differences among ethnic Han and Kazak women of Northwestern Chin (2018) PeerJ6:e4335; DOI 10.7717/peerj.4335

Evans, J., Heron, J., Francomb , H., Oke, S., \& Golding, J.: Cohort study of depressed mood during pregnancy and after childbirth. BMJ (2001) 257-60

Grupe, D. W., \& Nitschke, J. B.: Uncertainty and anticipation in anxiety: An integrated neurobiological and psychological perspective. Nat Rev Neurosci (2013)14:488-501

Guardino CM, Dunkel Schetter C. Coping during pregnancy: A systematic review and recommendations. Health Psychol Rev (2014) 8:70-94

Kantrowitz-Gordon, I.: Internet confessions of Postpartum Depression. Issues in Mental Health Nursing, (2013) 874-882.

Kaplan, B., \& Sadock, A.: Synopsis of Psychiatry, Behavioral Sciences/Clinical Psychiatry. 10th ed. Philadelphia: Lippincott Williams and Wilkins (2010)

Laggford, J.: The Role of Family Support in an Integrated Early Childhood System. Helping Families Get What They Need to Support They Need to Support Support Their Children's Development. (2009) Center for the Study of Social Policy. US

Lusskin SI, Pundiak TM, Habib SM.: Perinatal depression: Hiding in plain sight. Can J Psychiatry (2007) 52:479-88

Nemade, R., Reiss, S. N., \& Dombeck, M.: Psychology of DepressionPsychodynamic Theories (2011)

Oslen, M., Laursen, T. M., Mendelson, T., Pedersen, C. B., Mors, O., \& Mortensen, P. B.: Risk and predictors of readmission for a mental disorder during the postpartum period. Arch Gen Psychiatry (2009) Vol. 66: 189-98

Thurgood, S. M., Avery, D., \& Williamson, L.: Postpartum Depression. American Journal of Clincal Medicine (2009) Six. 17-22

Vigot, S. N., Villegas, L., Dennis, C. L., \& Ross, L. E.: Prevalence and risk factors for postpartum depression among women with preterm and low-birth weight infants: a systematic review. BJOG: An International Journal of Obstetrics and Gynaecology, 117, 540-550. doi: 10.1111/j.1471-0528 (2010) 02493.x 
